\section{Laurence Klotz}

Président

Association des urologues du Canada

president@cua.org

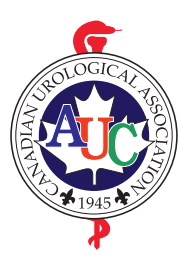

'AUC a été représentée avec fierté lors du Congrès de I'AUA en avril à Chicago. Pour la première fois, nous avions un kiosque dans la salle d'exposition et, selon les commentaires que notre personnel a reçus, les participants ont démontré beaucoup d'intérêt envers l'Association.

De plus, I'AUA a honoré 2 de nos membres en leur remettant des prix prestigieux. Tout d'abord, le docteur Mostafa Elhilali a reçu le tout-premier Prix pour I'ensemble de sa carrière décerné par I'AUA. Ce prix est un témoignage du profil remarquable du docteur Elhilali, et de son leadership au sein de la communauté urologique internationale. Quant au docteur J. Curtis Nickel, il a également été honoré en recevant le Certificat du Mérite, pour son immense contribution dans l'étude de la prostatite et de I'hyperplasie bénigne de la prostate. Le fait que ces prix aient été remis à des Canadiens prouve que l'AUA possède un degré remarquable de générosité et d'inclusion, et souligne l'étroite collaboration entre nos 2 pays dans le domaine de l'urologie. Cela indique également à quel point l'AUA tient l'urologie au Canada en haute estime. Ces hommes méritent nos félicitations les plus sincères.

Dans le dernier numéro du $J A \cup C^{1}$, j'ai discuté du Processus de planification stratégique de I'AUC, qui a résulté en un plan stratégique officiel que l'Exécutif de l'AUC a ratifié. Le plan prévoit de mettre en place des priorités pour les 5 prochaines années et comprend beaucoup d'éléments de mise en action et des points de repères pour la prochaine année, dont plusieurs nouvelles initiatives et directions pour l'AUC. Le document sera distribué à tous les membres de l'AUC dans un avenir rapproché. D'ailleurs, l'Exécutif de I'AUC aimerait recevoir vos commentaires à ce sujet. Nous espérons incorporer vos suggestions et présenter une version finale du document, en vue d'obtenir l'approbation des membres, lors du Congrès de Toronto en juin. Restez à l'écoute!

Par ailleurs, nous avons également plusieurs autres développements à vous annoncer. Nous avons établi un Comité de planification du congrès annuel afin d'assurer une continuité, de préserver une mémoire historique et de fournir des conseils aux organisateurs des congrès. Nous nous attendons à ce que ce groupe facilite la planification de notre Congrès annuel de manière significative. Nous avons également créé une nouvelle section en ligne " réservée aux membres » sur le site web de l'AUC. Nous vous encourageons à y jeter un coup d'œil — www.cua.org. Ce site comporte énormément de potentiel, dont l'annonce d'offres d'emploi et un panel de discussion.

La section du site web réservée aux membres a été mise en fonction en décembre 2008 pour permettre le paiement des frais de cotisation en ligne. Les membres peuvent également imprimer les Brochures info-patients et être mis au courant des annonces, pour les cours crédités d'EMC, et des annonces classées. À l'avenir, nous prévoyons présenter des modules de sondages ainsi qu'un répertoire des membres.

Et dernier point et non le moindre, nous anticipons le Congrès de l'AUC à Toronto avec impatience et entretenons beaucoup d'attentes à son endroit! En effet, il y a de tout pour tout le monde dans ce congrès: de nouvelles recherches, des mises à jour sur de nombreux sujets d'intérêt pour les urologues en exercice, des conférenciers qui provoquent la réflexion et nous inspirent, et un programme social formidable. Les enfants peuvent profiter d'un merveilleux camp de jour au Harbourfront ou d'un voyage à Canada's Wonderland. Nous avons cumulé ce que Toronto a de meilleur à offrir pour les partenaires: une visite au Club royal canadien du yacht sur l'île de Toronto, agrémentée d'une conférence par une célèbre voyageuse mondiale du domaine médical, l'occasion de se faire dorloter (toujours une bonne chose) à un spa de grand luxe, ainsi qu'une sortie au Royal Ontario Museum, ou le nouveau Musée des beaux-arts de l'Ontario, pour voir l'exposition des manuscrits de la Mer morte. Mme Ursula Klotz et moi-même espérons vous retrouver avec votre famille à Toronto!

\section{Référence}

1. Klotz L. Ipsa scientia potestas est. Can Urol Assoc J 2009;3:108-9. 